\title{
НЕЙРОЭНДОКРИННЫЕ РАССТРОЙСТВА У ПОДРОСТКА, ПЕРЕНЕСШЕГО НОВУЮ КОРОНАВИРУСНУЮ ИНФЕКЦИЮ COVID-19
}

\author{
Никитина И.Л., Саракаева Л.Р.
}

Федеральное государственное бюджетное учреждение «Национальный медицинский исследовательский центр им. В.А. Алмазова» Минздрава России, Санкт-Петербург, Россия

Актуальность. Несмотря на значительное увеличение опыта в отношении наблюдений клинических презентаций новой коронавирусной инфекции COVID-19, поражение эндокринной системы остается недостаточно изученным и требует дальнейших исследований. Широкая представленность в гипоталамусе и гипофизе рецепторов ангиотензинпревращающего фермента 2 типа (АПФ2) и клеточной трансмембранной сериновой протеазы типа 2 (TMPRSS2) определяет высокую тропность вируса SARS-COV2 к данным структурам мозга. При этом поражения гипоталамо-гипофизарной области вследствие перенесенной новой коронавирусной инфекции в детской популяции в настоящее время практически не изучены.

Цель. Описание и анализ клинического случая развития нейроэндокринных расстройств у подростка, перенесшего COVID-19.

Материалы и методы. Пациентка 13 лет, дважды перенесшая новую коронавирусную инфекцию COVID-19, прошла комплексное клинико-лабораторно-инструментальное обследование в условиях стационара.

Описание клинического случая и обсуждение. Девочка 13 лет, профессиональная спортсменка, дважды перенесла новую коронавирусную инфекцию, заболевание сопровождалось лихорадкой, выраженной головной болью, не купирующейся приемом анальгетиков. Спустя несколько месяцев после выздоровления отмечает нарастание слабости, значимое снижение толерантности к физическим нагрузкам, нарушение координации движений, в связи с чем обратилась к неврологу. При неврологическом обследовании выявлено нарушение проприоцептивной чувствительности, что характерно для поражения боковых рогов спинного мозга, назначена нейрометаболическая терапия.

С жалобами на сохраняющуюся выраженную слабость пациентка направлена к эндокринологу, по результатам гормонального обследования обнаружено выпадение адренокортикотропной, тиреотропной и гонадотропной функции гипофиза: уровень кортизола 73 нмоль/л (101.2 - 535.7), адренокортикотропного гормона (АКТГ) 17.76 пг/мл (7.2 - 63.3), свободного тироксина 8.8 пмоль/л (9.0 - 19.0), тиреотропного гормона (ТТГ) 1.965 мМЕ/л (0.350 - 4.940), лютеинизирующего гормона (ЛГ) 0.41 мМЕ/мл, фолликулостимулирующего гормона (ФСГ) 1.91 мМЕ/мл, эстрадиола 0.18 пг/мл. Была проведена проба с инсулиновой гипогликемией, по результатам которой отмечено отсутствие адекватной стимулированной секреции кортизола (максимальный выброс составил 284.7 нмоль/л), при этом стимулированная секреция соматотропного гормона (СТГ) оказалась сохранной - 15.52 
нг/мл. Проведена магнитно-резонансная томография (МРТ) гипофиза: данных за объемный процесс, геморрагические, ишемические изменения в хиазмально-селлярной области не получено, структура гипофиза не изменена, МР-признаков гипофизита нет.

Назначена заместительная гормональная терапия центрального гипокортицизма и гипотиреоза, при этом в течение месяца произошло спонтанное восстановление гонадотропной функции гипофиза, проявившееся стартом менархе.

По совокупности данных анамнеза и обследования можно предположить наличие у пациентки постинфекционного поражения центральной нервной системы (вариант нейроковида) с вовлечением в патологический процесс гипоталамо-гипофизарной системы и спинного мозга.

Выводы. Необходимо проявлять настороженность в отношении развития нейроэндокринных расстройств у детей, перенесших COVID-19; симптомы поражения эндокринной системы могут иметь неспецифический характер, что обуславливает позднюю диагностику заболевания.

КЛЮЧЕВЫЕ СЛОВА: гипопитуитаризм; новая коронавирусная инфекция COVID-19; подростки 